\title{
Electron cooling and the connection between expansion and flux-density evolution in radio supernovae
}

\author{
I. Martí-Vidal ${ }^{1}$, M. A. Pérez-Torres ${ }^{2}$, and A. Brunthaler ${ }^{1}$
}

\author{
1 Max-Planck-Institut für Radioastronomie, Auf dem Hügel 69, 53121 Bonn, Germany \\ e-mail: imartiv@mpifr-bonn.mpg.de \\ 2 Instituto de Astrofísica de Andalucía (CSIC) Apdo. Correos 2004, 08071 Granada, Spain
}

Received 9 November 2010 / Accepted 23 January 2011

\section{ABSTRACT}

\begin{abstract}
Radio supernovae (RSNe) are weak and rare events. Their typical maximum radio luminosities are of the order of only $10^{27} \mathrm{erg} \mathrm{s}^{-1} \mathrm{~Hz}^{-1}$. There are, however, very few cases of relatively bright (and/or close) RSNe, from which the expansion of the shock and the radio light curves at several frequencies have been monitored covering several years. Applying the standard model of radio emission from supernovae, it is possible to relate the defining parameters of the modelled expansion curve to those of the modelled light curves in a simple algebraic way by assuming an evolution law for the magnetic field and for the energy density of the population of synchrotron-emitting electrons. However, cooling mechanisms of the electrons may considerably affect this connection between light curves and expansion curve, and lead to wrong conclusions on the details of the electron acceleration and/or on the CSM radial density profile. We study how electron cooling modifies the flux-density decay rate of RSNe for a set of plausible/realistic values of the magnetic field and for different expansion regimes. We use these results to estimate the magnetic fields of different RSNe observed to date and compare them to those obtained by assuming energy equipartition between particles and magnetic fields. For some of the best monitored RSNe, for which deceleration measurements, optically thin spectral index, and power-law time decay have been observed (SN 1979C, SN 1986J, SN 1993J, and SN 2008iz), we find self-consistent solutions for the index of the power-law circumstellar density profile ( $s=2$ for all cases), the index of the power-law relativistic electron population (rather steep values, $p=2.3-3.0$ ) and the initial magnetic field (ranging from $\sim 20$ to $>100 \mathrm{G}$ ).
\end{abstract}

Key words. acceleration of particles - radiation mechanisms: nonthermal - radio continuum: stars - supernovae: general

\section{Introduction}

Radio supernovae (RSNe), which are the radio counterparts of core-collapse supernovae ( $\mathrm{SNe})$, are weak and rare events. Only about $10-20 \%$ of the observed $\mathrm{SNe}$ are detected in radio (e.g., Weiler et al. 2002). Moreover, their typical maximum radio luminosities are of the order of $10^{27} \mathrm{erg} \mathrm{s}^{-1} \mathrm{~Hz}^{-1}$ (flux densities of the order of $1 \mathrm{mJy}$ for extragalactic distances, close to the sensitivity limits of present detectors). There are, however, very few cases of relatively bright $\mathrm{RSNe}$, from which the expansion curve of the shock, using Very Long Baseline Interferometry (VLBI) observations, and radio light curves at several frequencies were obtained covering in some cases several years, e.g.: SN 1979C, SN 1986J, SN 1993J, and SN 2008iz. Although there are only a handful of objects, their detailed study allowed us to check and refine the current theoretical models of radio emission in supernovae. This small number of well-observed RSNe may also dramatically increase in the near future, thanks to the forthcoming ultra-sensitive interferometers with a high spatial resolution, like the Square Kilometre Array (SKA).

Using the standard model of radio emission from supernovae (Chevalier 1982a,b), it is possible to relate the defining parameters of the modelled expansion curve to those of the modelled light curves in a simple algebraic way by assuming an evolution law for the magnetic field (and for the density of the population of synchrotron-emitting electrons) and a radial density profile for the circumstellar medium, CSM (see, e.g. Weiler et al. 2002). The decay in the radio-light curves according to this model is related to the time decay in the magnetic field and the radial decay of CSM density. However, the continuous energy loss by the relativistic electrons (electron cooling), mainly owing to synchrotron radiation (i.e., radiative cooling), but also to adiabatic expansion and inverse-Compton scattering, are not considered in the derivation of this relation between light curves and expansion curve. Electron cooling may considerably affect the shape of the light curves for a given expansion curve. For instance, Martí-Vidal et al. (2011b) succesfully modelled the exponentiallike decay of the SN 1993J radio light curves at late epochs that were reported in Weiler et al. (2007), using only radiativecooling effects, and assuming that the density of the CSM was negligibly low at large distances to the progenitor star ${ }^{1}$. In any case, it seems clear that if electron cooling is not considered in the modelling of the radio light curves of a supernova, it could result in wrong estimates of the model parameters. In this paper, we study how electron cooling modifies the flux-density decay rate of RSNe for several values of the magnetic field and for different expansion regimes. These results can be used to estimate the magnetic fields of observed RSNe.

In the next section, we outline the standard model of radio emission from supernovae. In Sect. 3 we study the effect of electron cooling in the population of emitting electrons and in the flux-density decay rate. In Sect. 4 we present the results of several simulations of the expansion and radio light curves of RSNe.

\footnotetext{
${ }^{1}$ An additional effect caused by the escaping of the electrons from the emitting region may also be necessary to model the light curves of SN 1993J, were the density of the CSM not negligible at those large distances to the progenitor.
} 
In Sect. 5 we explain how these results can be used in real cases to estimate physical quantities in $\mathrm{RSNe}$, report estimates of the magnetic fields for several observed RSNe, and compare these estimates with those obtained by assuming particle-field energy equipartition. In Sect. 6 we summarize our conclusions.

\section{Connection between expansion and radio light curves in RSNe}

In the standard model of emission from supernovae (Chevalier 1982a,b), the spherically-symmetric expanding shock is described as a contact discontinuity plus two shocks, one moving backwards (from a Lagrangian point of view) and the other moving forwards, shocking the CSM. A fraction of shocked CSM electrons is accelerated to relativistic energies, possibly because of statistical Fermi processes, and produces synchrotron emission at radio wavelengths as they interact with high magnetic fields in the shocked CSM region.

The distance, $r$, from the contact discontinuity to the centre of the expansion evolves as a power-law of time $\left(r \propto t^{m}\right)$ with an expansion index, $m$, that depends on the radial density profiles of $\operatorname{CSM}\left(\rho_{\mathrm{CSM}} \propto r^{-s}\right)$ and ejecta $\left(\rho_{\mathrm{ej}} \propto r^{-n}\right)$ in the form (Chevalier 1982a,b)

$m=\frac{n-3}{n-s}$.

This solution of the shock expansion holds for $n>5$ and $s<3$. The structure of the shock (contact discontinuity plus backward and forward shocks) expands in a self-similar way. Therefore, the expansion of the forward and backward shocks also follows the law $\propto t^{m}$.

On the other hand, the distribution of relativistic electrons in energy space follows a power law $\left(N \propto E^{-p}\right)$ and the energydensity of the magnetic field is assumed to be proportional to the energy-density of the shock (i.e., $B^{2} \propto n V^{2}$, where $B^{2}$ is the average magnetic field squared, $n \propto r^{-s}$ is the particle number density, and $V \propto r^{(m-1) / m}$ is the shock expansion velocity). Hence,

$B \propto t^{m(2-s) / 2-1}$.

We need to look at a limitation in the standard model at this point. For a standard CSM particle density of $10^{8} \mathrm{~cm}^{-3}$ at a distance of $10^{15} \mathrm{~cm}$ from the explosion centre, and an expansion velocity of $20000 \mathrm{~km} \mathrm{~s}^{-1}$, a magnetic field of 50-60 G translates into a similar energy density for the expanding shock and the magnetic field. Such a large magnetic-field energy density may affect the hydrodynamics of the shock ${ }^{2}$. This effect is neglected in the model (which, indeed, assumes that the magnetic-field energy density is a small fraction of that of the shock). Hence, for the cases of very large magnetic fields reported in Sect. 5, high CSM particle densities and/or high expansion velocities may accordingly be considered to make the magnetic-field estimates consistent in the frame of the standard model.

The fraction of accelerated particles by the shock, or the injection efficiency of the shock, is also assumed to be proportional to the shock energy density. Under all these assumptions and considering that the intensity of synchrotron radiation is (e.g. Pacholczyk 1970)

$I \propto N B^{(1+p) / 2}$

\footnotetext{
2 Detailed magneto-hydrodynamic simulations would be necessary to study the real impact of large magnetic fields in the evolution of the expanding shock.
}

it is possible to derive the intensity, $I$, in the optically-thin regime if we neglect electron cooling. Since $\dot{N}(E) \propto E^{-p} n r^{2} V \mathrm{~d} t$, it can be shown that $I \propto v^{-\alpha} t^{\beta}$, with

$\alpha=\frac{p-1}{2}$,

and

$\beta=\frac{1}{4}(m(2(11+p)-(5+p) s)-2 p-10)$.

This equation establishes a direct relation between the decay index of the radio light curves in their optically-thin regime, $\beta$, on one hand, and the supernova expansion index, $m$, the energy index of the injected relativistic electrons, $p$, and the index of the CSM radial density profile, $s$, on the other hand. For the case of a constant pre-supernova mass-loss wind (i.e., $s=2$ ) this equation reduces to $\beta=(6 m-p-5) / 2$ (e.g., Weiler et al. 2002).

\section{Radiative and adiabatic cooling of the relativistic electrons}

The supernova shock is continuously accelerating electrons from the shocked CSM. These electrons are distributed as $N \propto E^{-p}$. However, the already shocked electrons, which are emitting synchrotron radiation, loose energy and, therefore, shift towards lower energies in the electron-energy distribution. Since the number of electrons is conserved, we can make use of the continuity equation in energy space, i.e.,

$\dot{N}=\nabla_{E}(N \dot{E})+S(E, t)-L(E, t)$,

where $S(E, t)$ is the source function (the new electrons continuously accelerated by the shock) and $L(E, t)$ accounts for the escaping of electrons from the emitting region. We will assume that $L(E, t)=0$ (in Martí-Vidal et al. 2011b, we use $L(E, t) \propto N$ to model the SN 1993J radio data, although the effects of this term are very small compared with $S(E, t)$ until very late epochs, when a large drop in the CSM density profile takes place). We show in Appendix A that the source function is $S(t) E^{-p}$, where

$S(t)=N_{0} F_{\text {rel }} \frac{p-1}{E_{\mathrm{m}}^{1-p}}\left(\frac{t}{t_{0}}\right)^{m(5-s)-3}$,

where $N_{0}$ is the number density of shocked CSM electrons at a reference epoch $\left(t_{0}\right), F_{\text {rel }}$ is the fraction of accelerated electrons (of the order of $10^{-5}$ for SN 1993J), and $E_{\mathrm{m}}$ is the minimum energy of the relativistic electrons (we set $E_{\mathrm{m}}=m_{\mathrm{e}} c^{2}$, although this value is not relevant in the optically-thin regime of the light curves).

The term $\dot{E}$ takes into account the energy loss (or gain) of the electrons. The energy loss can be either radiative, adiabatic, and/or caused by free-free interactions with atoms or ions in the CSM. The energy gain can come from self-absorption of the synchrotron radiation or from inverse-Compton scattering, although these effects are negligible in the optically-thin part of the light curve (and also for large magnetic fields), which is of interest here. For the case of radiative losses we have

$\dot{E}_{\mathrm{r}}=-c_{2} B_{\perp}^{2} E^{2}\left(\frac{t}{t_{0}}\right)^{m(2-s)-2}$,

where $c_{2}=2.37 \times 10^{-3}$ in cgs units (see Pacholczyk 1970), and $B_{\perp}$ is the magnetic field at a reference epoch $\left(t_{0}\right)$ averaged in the orthogonal planes to the electron trajectories. For a random 
distribution of magnetic-field lines and electron trajectories, $B_{\perp}$ is equal to $\sqrt{2 / 3}$ times the total averaged magnetic field, $B_{0}$, at the reference epoch. For the case of adiabatic losses we have

$\dot{E}_{\mathrm{a}}=\frac{1}{r} \frac{\mathrm{d} r}{\mathrm{~d} t} E=m \frac{E}{t}$.

Therefore, if radiative cooling and adiabatic expansion are the dominant processes of energy loss by the electrons, we have

$\dot{E}=-c_{2} B_{\perp}^{2} E^{2}\left(\frac{t}{t_{0}}\right)^{m(2-s)-2}-m \frac{E}{t}$,

in Eq. (10) we neglected the term that accounts for free-free interactions of the electrons with the surrounding CSM atoms and ions $\left(\dot{E} \propto r^{-s} E\right)$, because this term is much smaller than the radiative and adiabatic terms in the optically-thin regime of the light curves. In Appendix B we analyse under which conditions the free-free term is not negligible compared with the radiative and adiabatic terms.

Equation (6) (together with Eqs. (7) and (10)) is a typical difussion-like partial differential equation that can be numerically integrated using, for instance, a semi-implicit approach (e.g. Martí-Vidal et al. 2011b). However, since we neglect synchrotron self-absorption, inverse Compton, and free-free interactions (i.e., only the radiative and adiabatic terms in $\dot{E}$ are considered), it is also possible to find an integral form for the solution of this simplified version of Eq. (6). We show this solution in Appendix C.

From the numerical solution of $N(E, t)$, we can estimate the flux-density decay rate of the light curves, because the intensity is

$I \propto t^{m(2-s) / 2-1} \int_{E_{m}}^{\infty} N F(x) \mathrm{d} E$,

where the power-law of time is related to the decay of the magnetic field (see Eq. (2)), $x$ is the ratio between the observing frequency and the critical frequency at energy $E$, and $F(x)$ is

$F(x)=x \int_{x}^{\infty} K_{5 / 3}(z) \mathrm{d} z$

where $K_{5 / 3}(z)$ is a Bessel function of the second kind (e.g., Pacholczyk 1970). Then we can estimate $\beta$ from the time evolution of $I$ for different combinations of $m, p, s, B_{0}, N_{0}$, and $F_{\text {rel }}$, and compare the results with Eq. (5) to check the effect of electron cooling in the light curves.

\section{Effect of magnetic fields in the radio light curves}

We show in Figs. 1 and 2 the $\beta$ obtained from our simulations as a function of $m$ and $B_{0}$ (the magnetic field at the reference epoch $t_{0}=5$ days $)$ for six values of $p(2.0,2.2$, and 2.4 , in Fig. $1 ; 2.6$, 2.8, and 3.0, in Fig. 2) and for three values of $s$ (1.6, 2.0, and 2.4). We computed $\beta$ at $5 \mathrm{GHz}$ between 300 and 1000 days after the shock breakout. Different selections of frequencies and/or age ranges result in deviations in $\beta$ of a few $\%$ at most.

Since we compute $\beta$ in the optically-thin part of the radio light curves, $N_{0}$ is not really important in the simulations (changing this value would affect the opacity in the early supernova evolution). In our case, the important quantity would be $N_{0} F_{\text {rel }}$, which accounts for the number of relativistic electrons. Indeed, $N(E, t)$ only depends on $B_{0}$ regardless of a constant scaling factor defined by $N_{0} F_{\text {rel }}$. Therefore, the value of $N_{0} F_{\text {rel }}$ does not really affect the estimates of $\beta$. To ensure that this statement is correct, we checked that the values of $\beta$ derived from our simulations are only sensitive to changes in $B_{0}, m, p$, and $s$.

If radiative cooling is negligible (i.e., for low values of $B_{0}$ ), the $\beta$ computed from our simulations approaches the values computed from Eq. (5) for all combinations of $m, p$, and $s$. This is an expected result, because the adiabatic losses alone (which are $\propto E$ ) do not affect the power-law of the electron distribution (Pacholczyk 1970). However, as the magnetic field increases, $\beta$ decreases in absolute value (i.e., the light curves become flatter). This result is in principle non-intuitive, since one would expect the light curves to be steeper as the radiative cooling (i.e., the energy loss of the electrons) becomes more important. The light curves in the optically-thin stage are flatter for larger $B_{0}$, because the magnetic field decreases as the supernova expands (see Eq. (2)) and cooling effects (which are smaller for smaller magnetic fields) are consequently less important as time goes by. Thus, $\dot{N}$ assymptotically approaches the value without cooling as the supernova expands. As a consequence, $\dot{N} / N$ (which affects the value of $\beta$ ) takes a higher value if we consider radiative cooling. In Appendix D we show the mathematical details of this discussion.

The largest deviations of $\beta$ with respect to the cooling-free value (i.e., that of Eq. (5)) correspond in all cases to the smallest decelerations of the shock (i.e., values of $m$ close to 1) and/or to the steepest CSM radial density profiles (i.e., higher values of $s$ ).

For the case $s=2$ we can approximate the $\beta$ shown in Figs. 1 and 2 with the phenomenological equation

$\frac{\beta\left(B_{0}\right)}{\beta\left(B_{0}=0\right)}=\left(\frac{F_{1} /(1-m)}{B_{0}+F_{1} /(1-m)}\right)^{F_{2} /((1-m)(2 p-1))}$,

where $\beta\left(B_{0}\right)$ corresponds to a magnetic field $B_{0}$ and $\beta\left(B_{0}=0\right)$ is the same as given in Eq. (5) (i.e., with no radiative cooling considered). The parameters $F_{1}$ and $F_{2}$ take the values $7.725 \mathrm{G}$ and 0.184 , respectively. The maximum deviation between the $\beta$ computed from Eq. (11) and those shown in Figs. 1 and 2 (for $s=2$ ) is only $\sim 3.5 \%$.

\subsection{Changes in the spectral index}

If electron cooling is not considered, there is a direct relationship between $p$ and the spectral index $\alpha$ (see, Eq. (4)): $p=1+2 \alpha$. However, when electron cooling is taken into account, there is a flux of electrons towards smaller $E$, which increases the value of $\alpha$. This effect is more important as we increase the observing frequency. We must notice, however, that new electrons are continuously being injected in the emitting region, and their energy distribution is assumed to be always $\propto E^{-p}$, so this fraction of electrons is unaffected by cooling. Therefore, the effect of cooling in the spectral-index steepening is somewhat mimicked by the new electrons entering the shocked CSM. The integration of Eq. (6) takes into account this trade-off between electron cooling and the source function. In Figs. 3 and 4 we show the simulated spectral indices averaged between 300 and 1000 days after shock breakout and centred at $5 \mathrm{GHz}$. We show $\alpha$ for the same values of $p$ and $s$ as in Figs. 1 and 2.

We notice that radiative cooling is more important at higher energies, so the (effective) spectral index should slightly increase with the observing frequency. for instance, the difference between the spectral indices centred at $5 \mathrm{GHz}$ (which are higher) and those at $1.7 \mathrm{GHz}$ (which are lower) is typically $2-3 \%$ for magnetic fields of $10 \mathrm{G}$ and 5-6\% for magnetic fields of $100 \mathrm{G}$. 

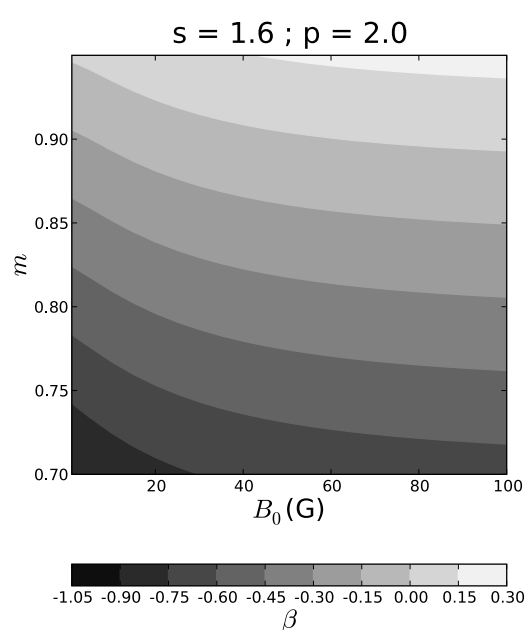
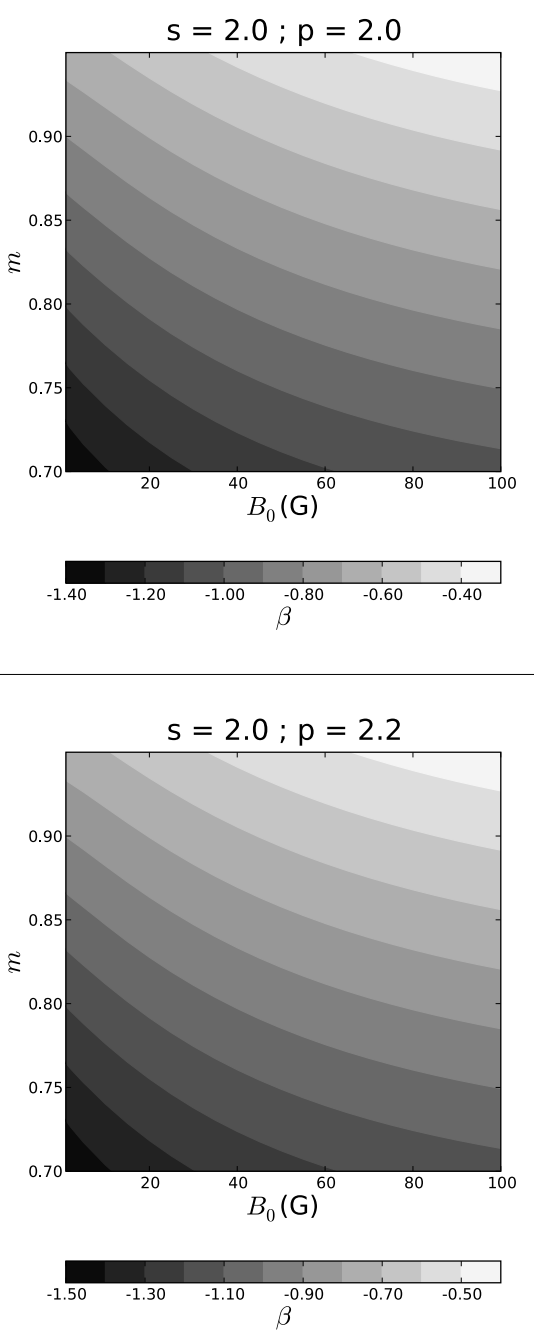

$s=1.6 ; p=2.2$

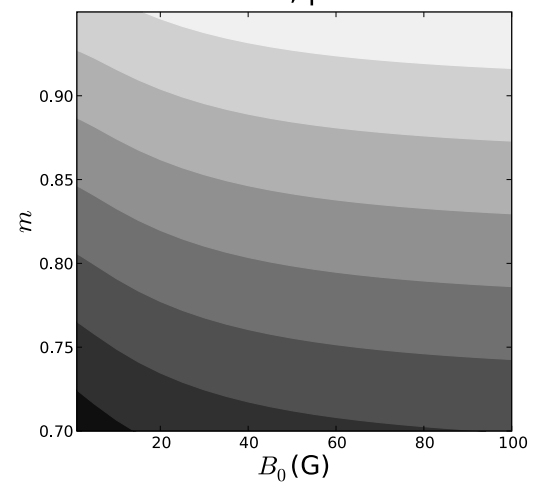

\begin{tabular}{cccccccccc}
\hline & & & & & & & & \\
-1.05 & -0.90 & -0.75 & -0.60 & -0.45 & -0.30 & -0.15 & 0.00 & 0.15
\end{tabular} $\beta$

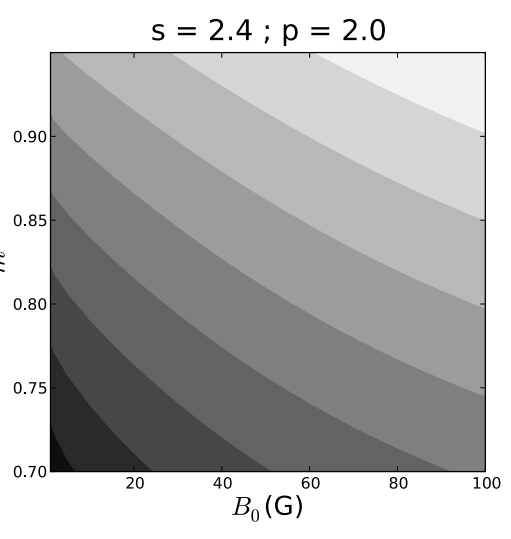

$-1.90-1.80-1.70-1.60-1.50-1.40-1.30-1.20-1.10-1.00$
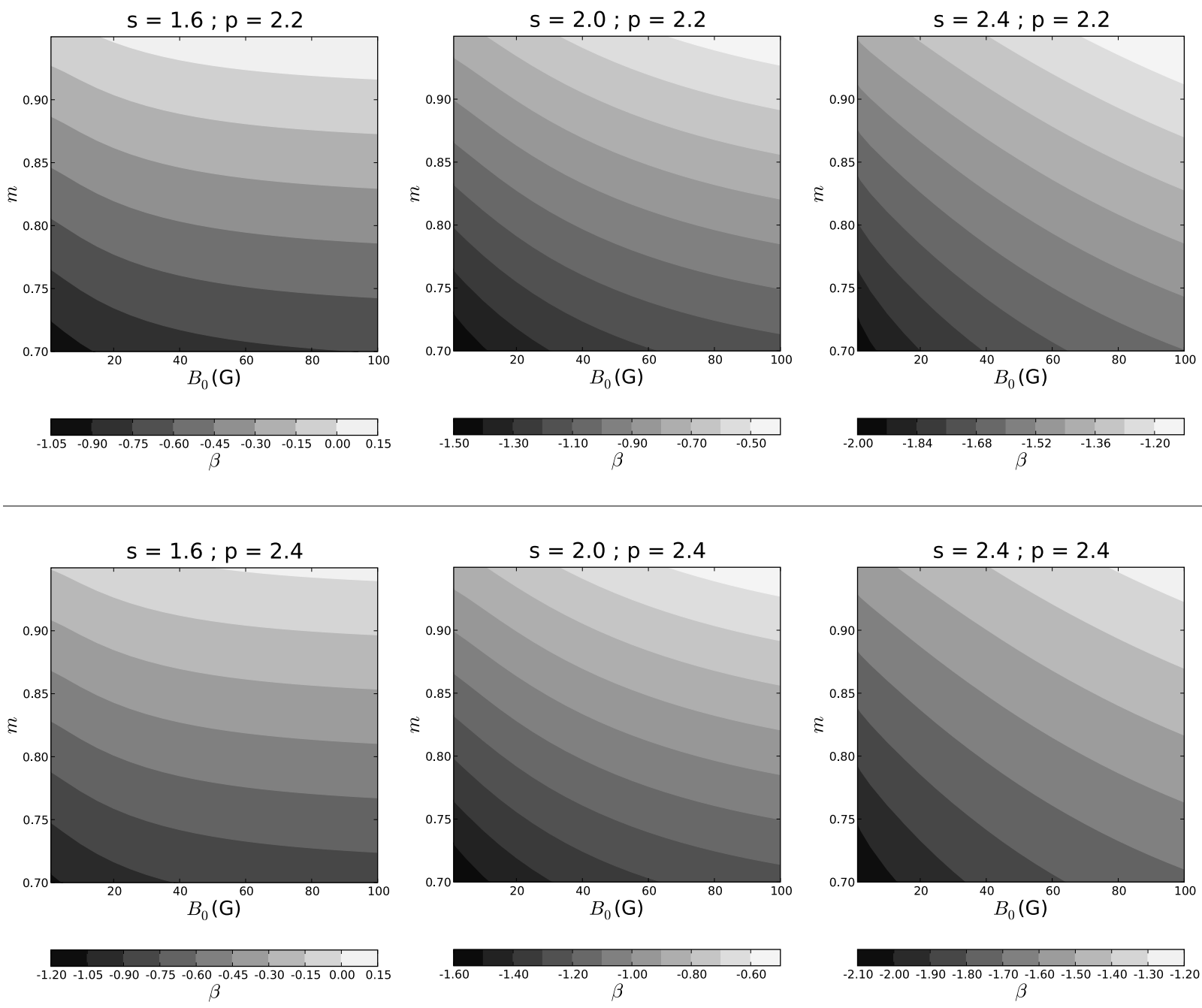

$\beta$

Fig. 1. $\beta$ as a function of $m$ and $B_{0}$ (reference epoch $t_{0}=5$ days) for a selection of values for $s$ and $p$. For $B_{0} \sim 0$, we roughly recover the $\beta$ given in Eq. (5). As we increase $B_{0}, \beta$ approaches 0 . This effect is more pronounced for higher values of $s$ (i.e., for steeper CSM radial density profiles).

The values of $\alpha$ obtained from our simulations tend to the expected values without cooling (i.e. $\alpha=(p-1) / 2$ ) when the magnetic field approaches 0 , also as expected. An increase in $B_{0}$ steepens the spectrum of the radiation (i.e., $\alpha$ increases) for all combinations of $s, m$, and $p$, because $\dot{E}_{\text {rad }} \propto E^{2}$.
For the case $s=2$ we can also approximate the $\alpha$ shown in Figs. 3 and 4 with a phenomenological equation

$$
\frac{\alpha\left(B_{0}\right)}{\alpha\left(B_{0}=0\right)}=\left(\frac{B_{0}}{F_{1}(2 m-1)(2 p+5)}+1\right)^{m /(2 p-1)} \text {, }
$$


I. Martí-Vidal et al.: Electron cooling in radio supernovae
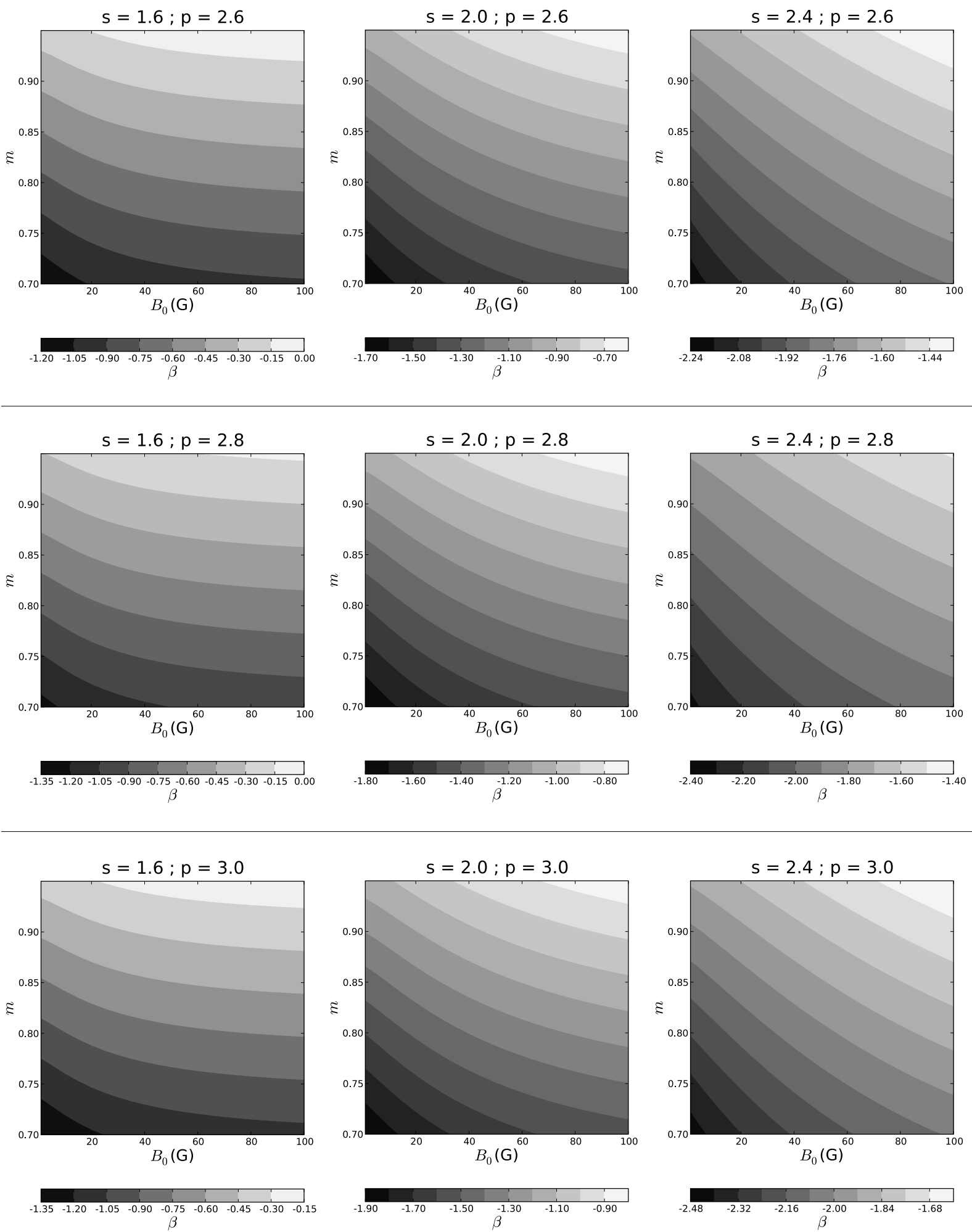

Fig. 2. Same as Fig. 1, but for a different set of values for $p$.

where $\alpha\left(B_{0}\right)$ corresponds to a magnetic field $B_{0}$ and $\alpha\left(B_{0}=0\right)$ is $(p-1) / 2$ (i.e., no radiative cooling considered). The parameter $F_{1}$ takes the value $3.04 \mathrm{G}$, and the maximum deviation between the $\alpha$ computed from Eq. (12) and those from the simulations is also $\sim 3.5 \%$ for all analysed values of $m, p$, and $B_{0}$.

Figures 1-4 (and eventually Eqs. (11) and (12)) can be used to estimate the magnetic field in a supernova by using the $\alpha, m$, and $\beta$ inferred from the observations (provided light curves and the expansion curve of the supernova have been observed). In the next section, we will estimate magnetic fields in a number of radio supernovae for which expansion curve and radio light curves are available.

\subsection{The special case $p=2$}

For the special case $p=2$ the effect of radiative cooling in the electron energy distribution should be negligible for all $E$, 

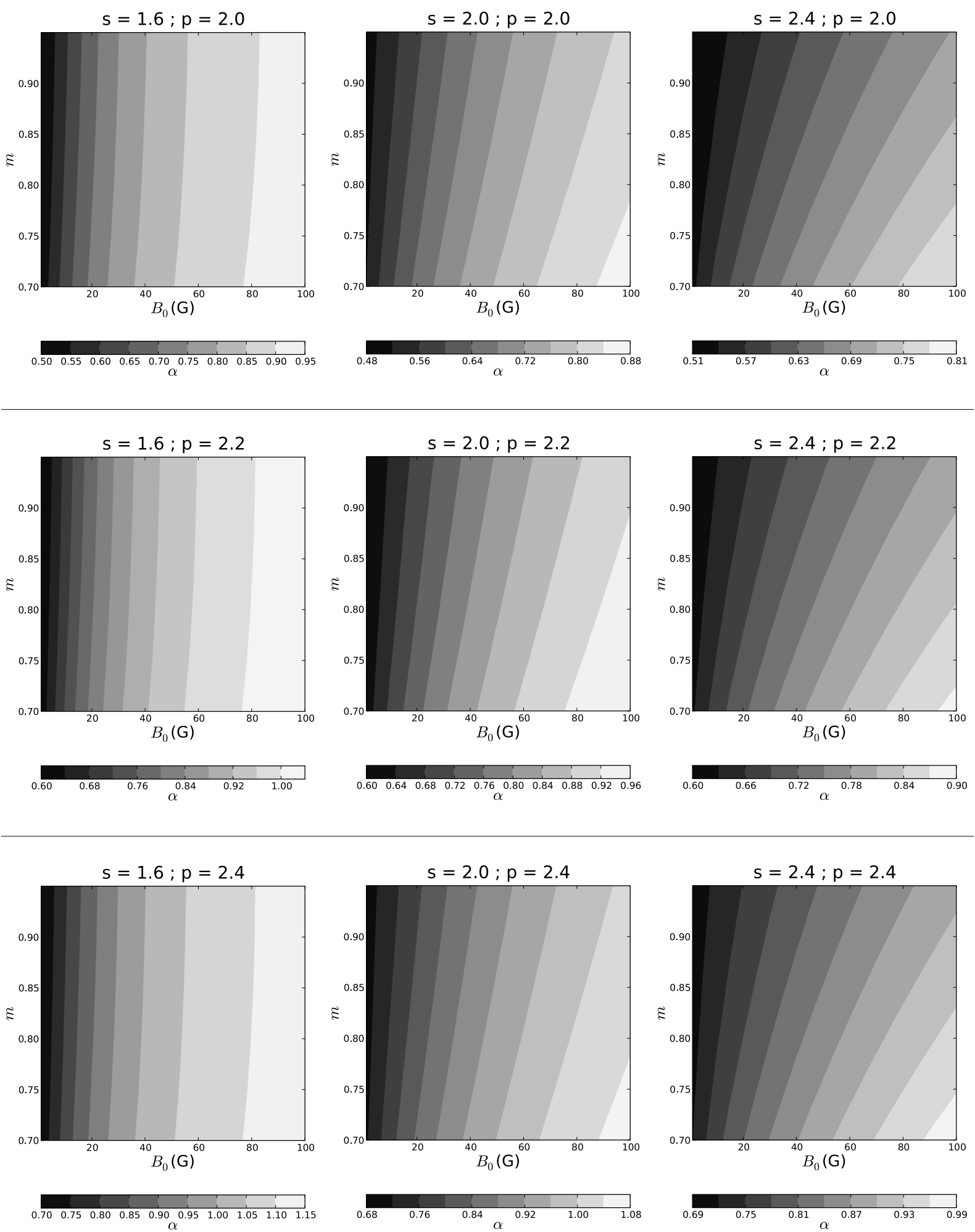

Fig. 3. Spectral index $\alpha$, centred at $5 \mathrm{GHz}$, as a function of $m$ and $B_{0}$ (reference epoch $t_{0}=5$ days) for a selection of values for $s$ and $p$. For $B_{0} \sim 0$ we roughly obtain the canonical value $\alpha=(p-1) / 2$. As we increase $B_{0}$, the spectra become steeper ( $\alpha$ increases). This effect is more pronounced for lower values of $s$ (i.e., for flatter CSM radial density profiles).

because $N \dot{E}_{\mathrm{r}}$ would not depend on $E$ and its contribution to the energy gradient of $N$ would therefore be null (see Eq. (6)). In principle, one would expect the population of electrons to evolve as if there were only adiabatic cooling, so neither $\beta$ nor $\alpha$ should depend on the magnetic field. However, when $p=2$, the evolution of $N(E, t)$ is not only determined by the source function,
$S(E, t)$, and the adiabatic term, but also by $t_{F}$ (see Appendix C, Eq. (C.6)), which is the time at which all electrons with energies higher than $E$ have energies below $E$ at time $t$. The time $t_{F}$ is longer than $t_{0}$ for high energies and/or long $t$ and depends on the magnetic field. Thus, even for $p=2$ the light curves and spectra will be somewhat modified by radiative electron cooling at high 
I. Martí-Vidal et al.: Electron cooling in radio supernovae
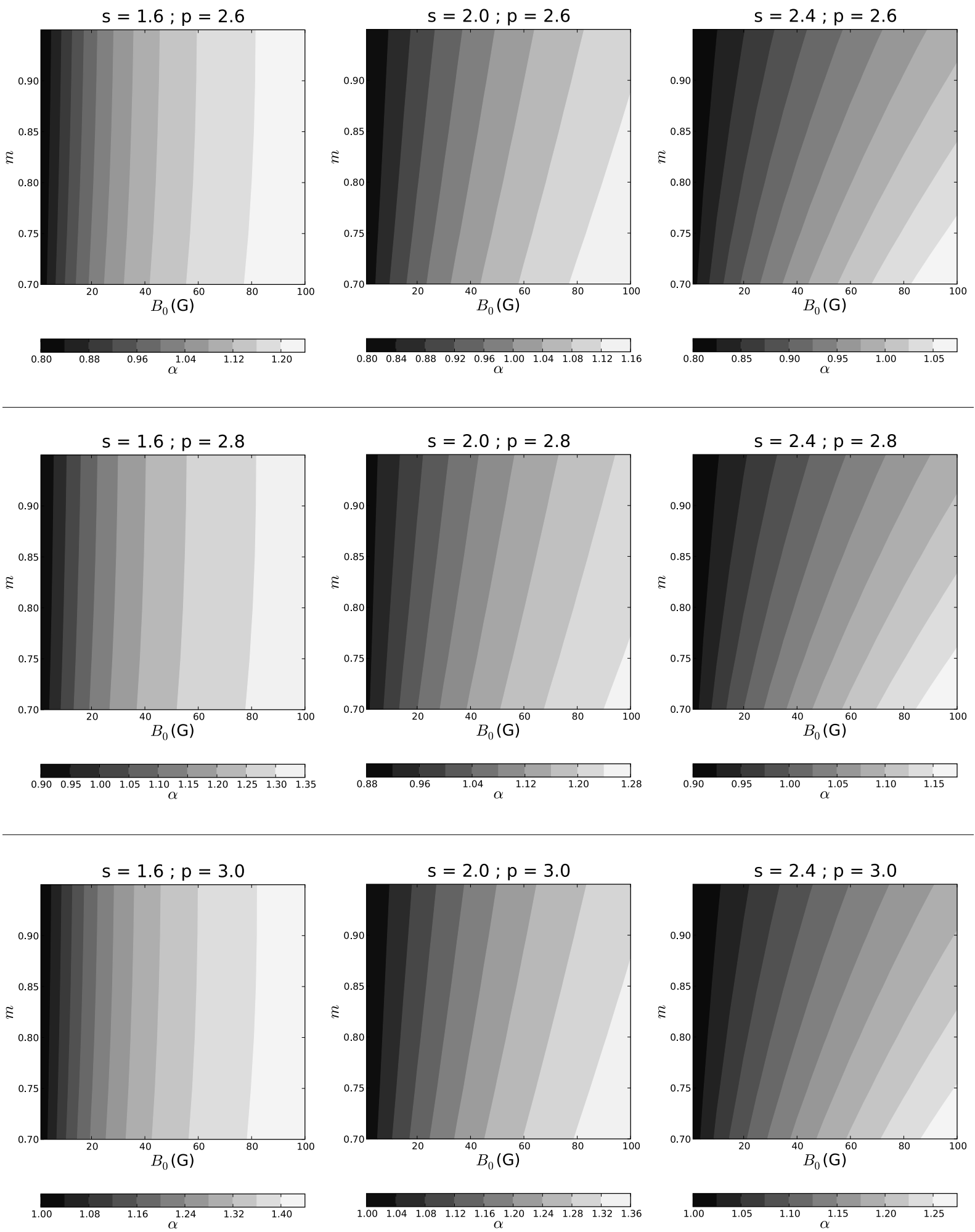

Fig. 4. Same as in Fig. 3, but for a different set of values for $p$.

frequencies and late epochs (those frequencies and supernova ages depend, of course, on the strength of the magnetic field and the deceleration of the shock), as shown in Figs. 1 and 3 (upper rows).

\section{Estimate of magnetic fields in observed RSNe}

If a radio supernova is strong enough to be monitored with VLBI, it is possible to estimate $m$ from the expansion curve and $\beta$ and $\alpha$ from the light curves ${ }^{3}$. If cooling is not considered, it is possible to derive $p$ from $\alpha$ and, using Eq. (5), it is possible

${ }^{3}$ We assume that all these quantities are determined in the opticallythin regime, which corresponds to a positive $\alpha$ (i.e., a steep spectrum) and a decreasing (or non-increasing) flux density, with the exception of very special cases ( $s \ll 2$ together with $m \sim 1$, see Figs. 1 and 2). In all observational cases studied here, the conditions for an optically-thin regime hold for all quantities used in our analysis. 
to derive $s$. Additionally, assuming a constant temperature of the CSM electrons, the opacity caused by free-free processes decreases as $t^{\delta}$, being $\delta=m(1-2 s)$ (e.g., Weiler et al. 2002). Therefore, if the light curves are well sampled in the opticallythick regime, another condition can be imposed on the parameters if we assume dominance of free-free absorption (FFA) over synchrotron self absorption (SSA). Self-consistency between all the parameters can then be checked.

However, it is unclear to which extent FFA dominates the light curves of usual RSNe. For instance, SSA has shown to be by far the dominant absorption mechanism in the whole evolution of the SN 1993J light curves (Fransson \& Björnsson 1998; Pérez-Torres et al. 2001; Martí-Vidal et al. 2011b). Moreover, different forms of electron cooling, as we showed in the previous section, affect the values of $\beta$ and $\alpha$ for a set of $m, s$, and $p$, depending on the strength of the amplified magnetic field. In this section, we will infer the values of magnetic fields of several RSNe based on the the values of $\alpha, \beta$ and $m$ estimated from the observations. An a priori value for $s$ and/or $p$ must be assumed however to estimate $B_{0}$ using this approach.

\subsection{SN $1979 C$}

Weiler et al. (1991) reported more than 10 years of observations of the SN 1979C radio light curves at 15,5 , and $1.4 \mathrm{GHz}$. These authors fit $\alpha=0.74_{-0.08}^{+0.05}$ and $\beta=-0.78_{-0.03}^{+0.02}$. In regard of the expansion curve, different results have been reported by different authors. Marcaide et al. (2002) reported a strong deceleration in SN 1979C and, from a more complete VLBI dataset, Bartel \& Bietenholz (2003) reported an almost free expansion (i.e., $m \sim 1$ ) for 22 years. More recently, Marcaide et al. (2009b) reanalysed their VLBI data and complemented them with new 1.6 GHz observations and the data from Bartel et al. These authors arrived at the conclusion that the expansion of SN 1979C was indeed almost free $(m=0.91 \pm 0.09)$ for over 25 years.

The fitted $\alpha$ is very close to the value corresponding to $p=$ 2.5 without radiative cooling. Therefore, we conclude that either the magnetic field is very small (and hence $\alpha \sim(p-1) / 2$ ), or $p$ is lower than 2.5. Assuming $p=2.2$ (or $p=2.4$ ) and $s=2$, we estimate from Fig. 1 a magnetic field of $\sim 20 \mathrm{G}$ (or $\sim 40 \mathrm{G}$ ) at day 5. There are no solutions neither for $s=1.6$ nor $s=2.4$. Now, from Fig. 3, the observed $\alpha$ and $m$ imply $B_{0} \sim 20-30 \mathrm{G}$ (for $p=2.2$ ) and $B_{0} \sim 5-10 \mathrm{G}$ (for $p=2.4$ ). Therefore, based on the radiative-cooling assumption, the magnetic field at day 5 should be between 20 and $30 \mathrm{G}$ if $p \sim 2$.2. Indeed, from Eqs. (12) and (11) we find self-consistent estimates of $\alpha$ and $\beta$ for $p=2.3$ and $B_{0}=30 \mathrm{G}$.

How do these estimates of $B_{0}$ compare to the equipartition magnetic field? For an energy equipartition between particles and fields, we can estimate the magnetic field in the radiating region provided the size and the total luminosity of the source are known. The expression used for this estimate is taken from Pacholczyk (1970):

$B_{\text {eq }}=\left(4.5 c_{12}(1+k) / \phi\right)^{2 / 7} R^{-6 / 7} L_{R}^{2 / 7}$,

where $c_{12}$ depends on the spectral index, $\alpha$, and on the frequency range in the spectrum integration. $\phi$ is the filling factor of the emitting region to a sphere $(0.66$ for a shell-like structure of $30 \%$ fractional width), $R$ is the source radius, $L_{\mathrm{R}}$ is the integrated radio luminosity, and $k$ is the ratio between the heavy particle energy density and the electron energy density. We do not know the details of the particle acceleration, and the efficiency of acceleration could depend on the particle mass. Hence, $k$ can vary
Table 1. Model parameters for several RSNe.

\begin{tabular}{|c|c|c|c|c|c|c|}
\hline \multirow[t]{2}{*}{ Supernova } & \multicolumn{3}{|c|}{ Observed } & \multicolumn{2}{|c|}{ Assumed } & \multirow{2}{*}{$\begin{array}{c}\text { Derived } \\
B_{0}(\mathrm{G})\end{array}$} \\
\hline & $m$ & $\alpha$ & $\beta$ & $s$ & $p$ & \\
\hline \multirow{2}{*}{ SN 1979C } & 0.91 & 0.74 & -0.78 & 2.0 & 2.5 & $\sim 0$ \\
\hline & & & & 2.0 & 2.3 & $20-30$ \\
\hline \multirow[t]{2}{*}{ SN 1986J } & 0.69 & 0.7 & -1.18 & 1.7 & 2.4 & $\sim 0$ \\
\hline & & & & 2.0 & 2.0 & $30-50$ \\
\hline $\mathrm{SN}_{1993 \mathrm{~J}^{a}}$ & 0.87 & 0.98 & -0.78 & 2.0 & 2.5 & $60-80$ \\
\hline SN 2008iz & $0.89^{b}$ & 1.08 & -1.43 & $\begin{array}{c}2 \\
24\end{array}$ & $\begin{array}{l}3 \\
26\end{array}$ & $\begin{array}{c}\sim 0 \\
\sim 100\end{array}$ \\
\hline
\end{tabular}

Notes. Observed refers to those obtained from the fitted expansion and radio light curves; Assumed and Derived refer to those obtained from comparison with the results shown in Figs. 1-4. Cases with two possible solutions are given in two rows, one row for each solution. ${ }^{(a)}$ Fit to data between days 300 and 1000 after explosion (see text). ${ }^{(b)}$ Derived from VLBI observations (Brunthaler et al., in prep.)

from 1 (case of a much higher acceleration efficiency of the electrons compared to the protons) to $m_{\mathrm{p}} / m_{\mathrm{e}} \sim 2 \times 10^{3}$ (case of a similar acceleration efficiency for electrons and protons).

Using the spectral index and flux densities given by Weiler et al. (1991), the expansion curve given by Marcaide et al. (2009b), and the distance to the host galaxy (M 100) given by Ferrarese et al. (1996) of $16.1 \mathrm{Mpc}$, we estimate $L_{\mathrm{R}}=1.6 \times$ $10^{33} \mathrm{erg} \mathrm{s}^{-1}$ at day 5 after explosion. Therefore, the equipartition magnetic field could range between 10 and $85 \mathrm{G}$ (for $k=1$ and $k=2000$, respectively). Our estimated $B_{0}$, assuming $p=2.3$ and $s=2$, corresponds to low-to-intermediate values of $k$, i.e. low-to-intermediate efficiency in the acceleration of ions.

The cooling-free prediction of $\beta$ (Eq. (5)) for $s=2$ and $p=2.5$ is consistent with the observed one if $m=0.99$ (i.e. for an essentially non-decelerated expansion), which is indeed compatible with the value of $m$ reported in Marcaide et al. (2009b) at a $1 \sigma$ level. This latter possibility would imply a very low magnetic-field energy density, compared with the energy density of the particles.

In Table 1 (row 1) we summarize the values of $s, p$, and $B_{0}$ discussed for this supernova.

\subsection{SN 1986J}

There are a number of peculiarities in the radio light curves of SN 1986J compared with those of other RSNe. The spectral index cannot be well fitted to a single value from 1.4 to $23 \mathrm{GHz}$ (Weiler et al. 1990). In the optically-thin part of the light curves, $\alpha=0.7 \pm 0.1$ between 5 and $15 \mathrm{GHz}$, but $\alpha=0.2 \pm 0.2$ between 15 and $23 \mathrm{GHz}$. Additionally, Bietenholz et al. (2004) reported the discovery of a compact source in the shell centre of SN 1986J with an inverted spectrum, and interpreted this source as the result of accretion onto a black hole or to a young pulsar nebula.

The best-fit parameters reported in Weiler et al. (1990) are $\alpha=0.67_{-0.08}^{+0.04}$ and $\beta=-1.18_{-0.04}^{+0.02}$, based on observations up to the year 1989. Bietenholz et al. (2002) reported a much lower $\beta$ for later epochs, which slightly depends on the observing frequency (ranging from -2.7 at $8.4 \mathrm{GHz}$ to -3.5 at $23 \mathrm{GHz}$ ). In this work we will use the $\alpha$ and $\beta$ obtained from the early epochs (i.e., those up to year 1989) and between 5 and $15 \mathrm{GHz}$.

With regard to the expansion curve, Bietenholz et al. (2010) reported $m=0.69 \pm 0.03$, a value much lower than those of the other RSNe observed with VLBI ( 0.8-0.9).

From the extrapolated size at day 5 , a distance to the host galaxy (NGC 891) of $8.4 \pm 0.5 \mathrm{Mpc}$ (Tonry et al. 2001), and using $\alpha=0.7$, we obtain an equipartition magnetic field between 14 
and $100 \mathrm{G}(k=1$ and 2000, respectively) using Eq. (13) for day 5 after explosion.

How do these estimates of $B_{0}$ compare with those that can be obtained with our approach? A spectral index of 0.7 can only be obtained with $p=2.4$ or lower. Inserting the lowest value, $p=2$, in Eqs. (11) and (12) (or Figs. 1 and 3) self-consistent values of $\alpha$ and $\beta$ with $B_{0}=30-50 \mathrm{G}$. Using $p=2.2$ and $s=2$, we estimate from Fig. 1 a magnetic field of $\sim 60 \mathrm{G}$ at 5 days after explosion. From Fig. 3 and assuming the same values for $s$ and $p$, a magnetic field of $\sim 10 \mathrm{G}$ is estimated. Both estimates are incompatible. For $s=2.4$ or $s=1.6$ we cannot obtain a coherent estimate of the magnetic field either; using now $p=2.4$, the observed $\alpha$ requires, of course, $B_{0} \sim 0$ and the observed $\beta$ can only be explained with our simulations if $s \sim 1.6$. Therefore, a compatibility between Figs. 1 and 3 is found for low values of the magnetic field and a fairly flat CSM radial density profile $(s \sim 1.6)$. Indeed, the cooling-free prediction of $\beta$ given by Eq. (5) (which is similar to the one with cooling considered if $B_{0}$ is very small) is equal to the observed one for $p=2.4$ and $s \sim 1.7$. Hence, we conclude that either $p=2, s=2$, and $B_{0}=30-50 \mathrm{G}$, or $s<2, p \sim 2.4$ and $B_{0} \sim 0 \mathrm{G}$, can explain the radio data for this supernova. In Table 1 (row 2) we summarize the values of $s, p$, and $B_{0}$ discussed for this supernova.

\subsection{SN 1993J}

This is the radio supernova with best-observed light curves and expansion curve (see Pérez-Torres et al. 2001; Bartel et al. 2002; Marcaide et al. 2009a; Weiler et al. 2007; Martí-Vidal et al. 2011a,b, and references therein).

Fitting their observed light curves (taken until $\sim 400$ days after explosion), Weiler et al. (2007) obtained $\alpha=0.81, \delta=$ -1.88 , and $\beta=-0.73$. Therefore, without considering electron cooling, we obtain $p=2.6$ from the fitted $\alpha$, and from the expansion index reported in Martí-Vidal et al. (2011b) at late epochs ( $m=0.87$ ), we obtain $s=1.6$. Applying now Eq. (5), we derive $\beta=-0.44$, which is inconsistent with the value fitted to the light curves $(\beta=-0.73)$.

However, if we decrease $m$ to 0.82 , we can obtain a selfconsistent solution for $\beta$ with Eq. (5). This seems to be a strong evidence of a CSM radial density profile with an index $s<2$. Also, Mioduszewski et al. (2001) simulated radio images and the radio light curves of SN 1993J without taking radiative cooling into account, and claimed that $s \sim 1.7$ provides the best fit to the data.

However, the evidence of $s<2$ from Eq. (5) and from the fit of the optically-thick part of the radio lightcurves holds as long as the temperature of the thermal CSM electrons is taken to be constant throughout the whole extent of the CSM (to be able to use $\delta=m(1-2 s)$ ), which is not likely to apply in the case of SN 1993J (Fransson \& Björnsson 1998; Martí-Vidal et al. 2011b). Additionally, more recent analyses of the X-ray data from SN 1993J also discard the models with $s<2$ (Nymark et al. 2009; Chandra et al. 2009).

From their simultaneous analysis of the complete light curves and expansion curve of SN 1993J, Martí-Vidal et al. (2011b) reported $B_{0}=65.1 \pm 1.6 \mathrm{G}$ and $p=2.59 \pm 0.01$ for $s=2$. It was also noted by these authors that using values of $s<2$ resulted in poor fits to the data. From a very time-limited set of flux-density measurements, Fransson \& Björnsson (1998) fitted a similar magnetic field for day 5 after explosion $\left(B_{0} \sim 68 \mathrm{G}\right)$ also using $s=2$, although they fitted a different energy index for the electron distribution $(p=2.1)$.
Which magnetic field do we obtain for SN 1993J with our approach? Opacity effects in the supernova ejecta may affect the spectral index and $\beta$ at different frequencies and for different times (Marcaide et al. 2009a; Martí-Vidal et al. 2011b). Therefore, in our approach we must use the values of $\beta$ and $\alpha$ fitted to the subset of data where these ejecta-opacity effects are minimum or non-existent, and not those fitted to the whole dataset. Using the $5 \mathrm{GHz}$ and $8.4 \mathrm{GHz}$ data of Weiler et al. (2007) from day 300 to day 1000 after explosion, we obtain $\beta=-0.78 \pm 0.05$ at $5 \mathrm{GHz}$ and $\beta=-0.79 \pm 0.08$ at $8.4 \mathrm{GHz}$. The average spectral index between 8.4 and $5 \mathrm{GHz}$ at these epochs is $\alpha=0.98 \pm 0.19$.

This spectral index implies $p=3.0$ or lower. For any value of $p$, neither $s=1.6$ nor $s=2.4$ yield self-consistent estimates of $B_{0}$ using our approach. This is an additional evidence of a CSM with $s=2$ for SN 1993J. Assuming now that $s=2$, we estimate from Eqs. (11) and (12) that $p \sim 2.4$ and $B_{0}=60-80 \mathrm{G}$.

In Table 1 (row 3 ) we summarize the values of $s, p$, and $B_{0}$ discussed for this supernova.

The range of values of $B_{0}$ estimated this way agrees with the estimates reported in Fransson \& Björnsson (1998) and Martí-Vidal et al. (2011b). Nevertheless, here we used a subset of the observed light curves to avoid the undesired contribution of ejecta-opacity effects in our rough radiative-cooling model.

Fransson \& Björnsson (1998) and Martí-Vidal et al. (2011b) also discussed the particle-field energy equipartition based on their fitted magnetic fields. In both papers, the conclusion is that to obtain energy equipartition, an acceleration efficiency of the ions similar to that of the electrons (i.e., $k \gg 1$ in Eq. (13)) should take place in the shock.

\subsection{SN2008iz}

Marchili et al. (2010) reported a $5 \mathrm{GHz}$ light curve for this supernova, taken with the Urumqi telescope. Brunthaler et al. (2010) reported VLBI observations from which the explosion date and the expansion velocity could be estimated. Marchili et al. (2010) estimated an equipartition magnetic field between $0.3 \mathrm{G}$ and $2.1 \mathrm{G}$ (for $k=1$ and $k=2000$, respectively) at day 63 after explosion. Assuming $s=2$ (i.e., $B \propto t^{-1}$ ), this results in a magnetic field between 3.8 and $26.5 \mathrm{G}$ at day 5 after explosion.

If we use our approach, the spectral index, $\alpha=1.08 \pm 0.08$ (Marchili et al. 2010; Brunthaler et al. 2010), is compatible with $p \sim 3$ or lower. However, using $\beta=-1.43 \pm 0.05$ (Marchili et al. 2010) and $m \sim 0.89$ (derived from a set of VLBI observations; Brunthaler et al., in prep.), we find a self-consistent magnetic field of $\sim 100 \mathrm{G}$ for $p=2.6$ and $s=2.4$ (see Figs. 2 and 4), much larger than that reported in Marchili et al. (2010).

However, if $p \sim 3$, the magnetic field would be close to $0 \mathrm{G}$, regardless of the value of $s$ (in order to explain the spectral index). Now, if we set $s=2$, we obtain $\beta \sim-1.3$ for $m=0.89$. This value is close to, but lower than, the observed one, and would increase if $s$ would be slightly larger than 2 . Indeed, the uncertainties in $m, \alpha$, and $\beta$ can still make $s=2$ possible for $p \sim 3$. In any case, the magnetic field in the emitting region can be arbitrarily small if $p \sim 3$, and we cannot favour either this possibility or the estimate of $B_{0} \sim 100 \mathrm{G}$ obtained for $p=2.6$.

In Table 1 (row 5) we summarize the values of $s, p$, and $B_{0}$ discussed for this supernova.

\subsection{Other RSNe}

For RSNe where only the radio light curves are available, it is still possible to infer some information on magnetic fields and 
density structure of the CSM and/or ejecta, although with several additional assumptions. In this section, we study two cases that we consider more interesting than other typical RSNe.

\subsubsection{Radio transient in M 82}

The discovery of a new transient in M 82 has been recently reported in Muxlow et al. (2009), and a light curve with a practically constant flux density has been reported in Muxlow et al. (2010), with an spectral index of $\sim 0.7$. Indeed, looking at their Fig. 2, the flux density at $1.6 \mathrm{GHz}$ seems to be slightly increasing. If this transient in the starburst galaxy M 82 is a supernova, it would be a very special case, because $\beta \sim 0$. It is not possible to obtain this value of $\beta$ unless $s<2$ (see Eq. (5)), because the highest value of $m$ is 1 and $p$ is assumed to be larger than 1 . Indeed, from Eq. (5) we obtain $s \sim 0.6$, for $p=2$, and $s \sim 1.3$, for the extreme case $p=1$. Therefore, a plain light curve is a strong evidence of a CSM density profile much shallower than the canonical case $s=2$. In any case, another condition for $\beta \sim 0$, regardless of the strength of the magnetic field, is that $m \sim 1$ (see Fig. 1). Therefore, two clear conclusions can be extracted for this transient, provided it is a supernova: 1) the index of the CSM density profile is $s<2$ and 2) the deceleration index must be $m \sim 1$. Both conclusions imply that the index of the ejecta density profile, $n$, must be very large ( $n=20$, or even higher, see Eq. (1)). With regard to the spectral index, we conclude from Figs. 3 and 4 that the magnetic field would be up to $B_{0} \sim 20 \mathrm{G}$, assuming $s=1.6$ and $p=2$, and lower for larger $p$.

\subsubsection{SN 2000ft}

Supernova SN 2000ft was discovered by Colina et al. (2001). Pérez-Torres et al. (2009) presented an eight-year long radio monitoring of this supernova, which is located in the circumnuclear starburst of NGC 7469 (a luminous infra-red galaxy, LIRG, at a distance of $70 \mathrm{Mpc}$; Sanders et al. 2003). Pérez-Torres et al. (2009) followed the approach of Weiler et al. (2002) to fit the evolution of the radio light curves, using a standard value of $s=2$ for the CSM. This analysis resulted in a value for the spectral index $\alpha=1.27$ and a power-law time decay index $\beta=-2.02$. In addition, they also needed to include a foreground absorber, likely an H II region, to account for the non-detection of radio emission at frequencies around and below $1.7 \mathrm{GHz}$, in agreement with the observations reported by Alberdi et al. (2006).

While the value of $\alpha$ reported for SN 2000ft is not surprising, the value of $\beta$ is much higher (in absolute value) than those typically found in RSNe. From Eq. (5), we obtain values of $\beta$ similar to that of SN 2000ft if $s>2$ (see Figs. 1 and 2), although a low value of $m$ (together with a large $B_{0}$ ) or a large value of $p$ is also necessary to simultaneously explain the steep spectrum (see Fig. 4). If $s=2$, it is also possible to obtain a self-consistency between $\alpha$ and $\beta$, provided $B_{0} \sim 0, p=3.54$, and $m=0.75$.

In any case, we find that SN $2000 \mathrm{ft}$ should be a highly decelerated supernova ( $m$ between 0.7 and 0.8 ), the CSM density index should be $s=2$ or higher, and the energy distribution of the electrons must be quite steep ( $p=3$ or higher).

\section{Conclusions}

We showed the impact of energy losses of relativistic electrons in RSNe and their effect on the flux-density decay rate of the light curves in the optically-thin regime for different values of the magnetic fields and for different expansion curves.

If the magnetic-field energy density and the acceleration efficiency of the shock scale with the shock energy density, which is very likely the case for RSNe, we find that there is a tight relation between expansion index, $m$, spectral index, $\alpha$, and (optically thin) flux-density decay index $\beta$.

This connection between expansion and flux-density evolution in RSNe can be used to estimate the magnetic field of observed RSNe ( $B_{0}$ at a reference epoch) as well as its evolution with time for an assumed CSM radial density profile and energy index, $p$, of the relativistic electrons.

For a number of well observed RSNe (e.g., SN 1993J in M 81) we found self-consistent solutions for $B_{0}, m, s$, and $p$. A standard CSM density profile (i.e., $s=2$ ) can explain all observations, although we found evidence of non-standard values of $s$ for SN 1986J and SN 1979C. The index of the relativistic electron population takes fairly high values $(p=2.3-3.0)$, and the range of magnetic fields between all cases is large $\left(B_{0} \sim 20-100 \mathrm{G}\right)$. These strong magnetic fields imply effective amplification mechanisms in the radio-emitting region, which may be related to plasma turbulence (see, e.g., Gull 1973, or Jun $\&$ Norman 1995, and references therein).

Previous analyses of the radio light curves and expansion curves of these RSNe did not take into account the correct coupling between $m, \beta$, and $\alpha$ for different magnetic fields. Some of the results previously reported for these supernovae could, therefore, be internally inconsistent.

The magnetic fields obtained with our approach are in similar to the equipartition magnetic fields. For SN 1979C and SN 1986J we obtain a range of self-consistent magnetic fields similar to those derived from equipartition with a lower acceleration efficiency for ions (i.e., low-to-intermediate values of $k$ in Eq. (13)). Additionally, for SN 1986J there is evidence of $s<2$, provided the magnetic field is low. For SN 2008iz, either a very low magnetic field (with $s \sim 2$ ) or an extremely large magnetic field (with $s>2$ ) are necessary to model the light curve, given the high flux-density decay rate $(\beta=-1.43)$. For SN 1993J we obtain a magnetic field similar to that reported in Fransson \& Björnsson (1998) and Martí-Vidal et al. (2011b) although we use in our approach a subset of flux-density observations (and not the whole data set), to avoid possible biasing effects coming from the ejecta opacity (Martí-Vidal et al. 2011b).

For the RSNe that will be detected in the future (the high sensitivity of the forthcoming radio observatories, like ALMA and SKA, will allow the detection and monitoring of many other $\mathrm{RSNe}$ ), it will be necessary, as our results show, to study the connection between their expansion and flux-density evolution to obtain self-consistent results for the CSM profile, the electron energy index, and the magnetic field based on the observed spectral index, the expansion curve, and the flux-density decay rate.

Acknowledgements. I.M.V. is a fellow of the Alexander von Humboldt foundation in Germany. M.A.P.T. acknowledges support by the Spanish Ministry of Education and Science (MEC) through grant AYA 2006-14986-C02-01, and by the Consejería de Innovación, Ciencia y Empresa of Junta de Andalucía through grants FQM-1747 and TIC-126.

\section{Appendix A: Derivation of the source function of relativistic electrons}

The source function is related to the acceleration of part of the electrons from the CSM, because they interact with the expanding supernova shock. If we assume that the acceleration efficiency scales with the energy density of the shock (see Fransson \& Björnsson 1998, for a discussion of different possibilities and how they fit to the observations of SN 1993J), the density of electrons instantaneously accelerated at a given time, $t$, will be

$n_{\mathrm{rel}} \propto n_{\mathrm{cs}} V^{2}$, 
where $V$ is the velocity of the expanding supernova shock and $n_{\mathrm{cs}}$ is the density of the recently-shocked CSM, both quantities computed for the same time $t$. Since the radius of the shock is $r \propto t^{m}$, the number of relativistic electrons injected between $r$ and $r+\mathrm{d} r$ is

$N_{\text {rel }}=F_{\text {rel }} n_{\mathrm{cs}}^{0}\left(\frac{r}{r_{0}}\right)^{-s} V^{2} 4 \pi r^{2} \mathrm{~d} r$

where $s$ is the index of the CSM radial density profile, $n_{\mathrm{cs}}^{0}$ and $r_{0}$ are the CSM density and shock radius at a given reference epoch, $t_{0}$, and $F_{\text {rel }}$ is the acceleration efficiency (or fraction of CSM electrons that are accelerated) at the same epoch $t_{0}$.

These electrons are distributed according to $N \propto E^{-p}$ (with $E$ running from $E_{\min }=m_{\mathrm{e}} c^{2}$ to infinity, being $m_{\mathrm{e}}$ the electron mass). Therefore, the conservation of the number of electrons implies

$N_{\text {rel }}=K \int_{m c^{2}}^{\infty} E^{-p} \mathrm{~d} E$.

The factor $K$ accounts for the normalization of the electron energy distribution. This factor is

$K=\frac{p-1}{E_{\min }^{1-p}}$.

Hence, the source function (i.e., the energy distribution of electrons shocked between $r$ and $r+\mathrm{d} r$ ) is

$S(E, r)=F_{\text {rel }} N_{\mathrm{cs}}^{0} \frac{p-1}{E_{\min }^{1-p}}\left(\frac{r}{r_{0}}\right)^{5-s-3 / m} E^{-p}$,

which, in terms of time (given that $r / r_{0}=\left(t / t_{0}\right)^{m}$ ), reduces to Eq. (7).

\section{Appendix B: Radiative and adiabatic energy loss vs. free-free loss in RSNe}

The rate of energy loss caused by synchrotron radiation and adiabatic expansion, $\dot{E}_{\mathrm{r}}$ and $\dot{E}_{\mathrm{a}}$, are given in Eqs. (8) and (9), respectively. With regard to the energy loss caused by free-free interactions with the CSM, we have (Pacholczyk 1970)

$\dot{E}_{\mathrm{f}} \sim f_{1} n_{\mathrm{cs}} E$,

where $f_{1} \sim(1-8) \times 10^{-16}$ in cgs units. The exact value depends on the level of ionization of the nuclei in the CSM (lower values of $f_{1}$ correspond to higher levels of ionization, which are expected in the shocked CSM ${ }^{4}$. Since an electron with energy $E$ in interaction with a magnetic field $B$ emits synchrotron radiation mostly at its critical frequency (given by $v \sim c_{1} B E^{2}$, where $c_{1}=6.27 \times 10^{18}$ in cgs units, Pacholczyk 1970), the ratio of radiative loss to free-free loss for electrons emitting at the critical frequency $v$ is

$\frac{\dot{E}_{\mathrm{r}}}{\dot{E}_{\mathrm{f}}}=\frac{c_{2}}{f_{1}} \sqrt{\frac{v}{c_{1}}} \frac{B_{0}^{3 / 2}}{n_{0}}\left(\frac{t}{t_{0}}\right)^{(m(6-s)-6) / 4}$,

where we used the time evolution of $B$ given in Eq. (2). On the other hand, the ratio of diabatic to free-free energy loss for electrons emiting at the same frequency is

$\frac{\dot{E}_{\mathrm{a}}}{\dot{E}_{\mathrm{f}}}=\frac{m}{f_{1} n_{0} t_{0}^{m s}} t^{m s-1}$.

4 There is an additional contribution to $\dot{E}_{\mathrm{f}}$ that can be written as a modifying factor of $f_{1}$, which depends on $\log E$. We opted to neglect this small correction.
The ratios in Eqs. (B.2) and (B.3) evolve as power laws of time, whose indices depend on $s$ and $m$. Therefore, for some combinations of $s$ and $m$, the ratios will grow with time (and radiative and adiabatic losses will dominate over free-free losses), but for other combinations the ratios will decrease with time, and freefree losses may be comparable with the other contributions at late times.

For radiative vs. free-free losses, the time index in Eq. (B.2) is positive if

$m>\frac{6}{6+s}$

which implies values of $m$ higher than 0.75 for the canonical case $s=2$, although slightly higher values of $m$ for lower $s$ (for instance, $m>0.78$ if $s=1.6)$. However, even if $m$ is lower than these values (so the ratio $\dot{E}_{\mathrm{r}} / \dot{E}_{\mathrm{f}}$ decreases with time), radiative losses will still be higher than free-free losses for the times and observing frequencies of interest. For instance, with an initial magnetic field as low as $B_{0}=10 \mathrm{G}$ (at day 5 after explosion!) and a CSM density as high as $n_{0}=10^{9} \mathrm{~cm}^{-3}$, the ratio is $\sim 15$ at day 1000 after explosion, for $m=0.70$, observing at $5 \mathrm{GHz}$.

The ratio of adiabatic losses to free-free losses increases with time if $m>1 / s$. This relation allows expansion indices as low as 0.63 if $s=1.6$, and even lower values for higher $s$. Even in the (very special) cases where we would find $m<1 / s$, adiabatic losses would still be higher than free-free losses for the times and observing frequencies of interest. For instance, if $m=0.59$ (i.e., the minimum possible value compatible with the Chevalier model for $s=1.6$ ) and $n_{0}$ is as large as $10^{9} \mathrm{~cm}^{-3}$, this ratio is $\sim 10$ at day 1000 after explosion observing at $5 \mathrm{GHz}$.

In any case, for strongly decelerated RSNe (i.e., with low $m$ ) free-free losses may not be completely negligible, depending also on the CSM density (higher density implies larger free-free energy losses). In these special cases, the connection between $\beta$ and $m$ may not only depend on $s, p$, and $B_{0}$, but also on $n_{0}$. Hence, Eq. (6) will have to be individually integrated for each of these cases.

\section{Appendix C: Integral solution of the continuity equation in energy space}

The energy loss of an electron with energy $E$ at time $t$ is given in Eq. (10). Here we rewrite the equation,

$\dot{E}=-c_{2} \frac{2}{3} B_{\mathrm{ref}}^{2}\left(\frac{t}{t_{\mathrm{ref}}}\right)^{\rho} E^{2}-m \frac{E}{t}$,

where $\rho=2(m-s)-2$ and $B_{\text {ref }}$ is the magnetic field at a reference epoch $t_{\text {ref }}$ (we do not use the subindex 0 , to distinguish the reference epoch from the initial time of integration, $t_{0}$, see below). The solution to this equation is

$E(t)=\frac{(1+\rho-m) t_{0}^{m} E_{0}}{(1+\rho-m) t^{m}+2 / 3 c_{2} B_{\mathrm{ref}}^{2} E_{0} t_{\mathrm{ref}}^{-\rho}\left(t^{1+\rho} t_{0}^{m}-t_{0}^{1+\rho} t^{m}\right)}$,

where we computed the integration constant by assuming $E=$ $E_{0}$ at $t=t_{0}$ (i.e., the initial time of integration). We now write $E_{0}$ in terms of $E$ :

$E_{0}=\frac{(m-1-\rho) E}{(m-1-\rho)\left(t_{0} / t\right)^{m}+2 / 3 c_{2} B_{\mathrm{ref}}^{2} E t_{\mathrm{ref}}^{-\rho}\left(t^{1+\rho}\left(t_{0} / t\right)^{m}-t_{0}^{1+\rho}\right)}$.

At a given time, $t_{0}$, the electrons being shocked (let us call them $N^{0}\left(E_{0}\right)$ ) are distributed as $S\left(t_{0}\right) E_{0}^{-p}$ (where $S\left(t_{0}\right)$ is given in 
Eq. (7)). Since the number of electrons is conserved, at a later time, $t$, these electrons (i.e., not all the electrons, but just those shocked at time $t_{0}$ ) will have the distribution $N^{0}(E)$ given by

$N^{0}(E) \mathrm{d} E=N^{0}\left(E_{0}\right) \mathrm{d} E_{0} \rightarrow N^{0}(E)=N^{0}\left(E_{0}\right) \frac{\mathrm{d} E_{0}}{\mathrm{~d} E}$.

Applying Eq. (C.3), and its derivative, to Eq. (C.4), we obtain

$$
\begin{aligned}
& N^{\tau}(E)= \\
& \quad \frac{S(\tau)(m-1-\rho)^{2-p} E^{-p}(\tau / t)^{m}}{\left((m-1-\rho)(\tau / t)^{m}+2 / 3 c_{2} B_{\mathrm{ref}}^{2} E t_{\mathrm{ref}}^{-\rho}\left((\tau / t)^{m} t^{\rho+1}-\tau^{1+\rho}\right)\right)^{2-p}},
\end{aligned}
$$

where $t_{0}$ has been replaced by $\tau$, which can take any value in the evolution time of the supernova. A similar approach was described in Pacholczyk (1970, see his Sect. 6.3), although a constant and homogeneous magnetic field was used. Equation (C.5) is physically meaningful only when the power in the denominator is that of a positive number. Let us call $t_{F}$ the value of $\tau$ in a way that the denominator of Eq. (C.5) vanishes. For a given supernova, this value depends on $E$ and $t$. For larger $t$, all the electrons shocked at time $\tau$ will have energies below $E$. In other words, no electrons shocked at time $t_{F}$ (and earlier times) contribute to the electron distribution at time $t$ for energies above $E$.

It is now straightwforward to conclude that the total number of electrons at time $t$ and energy $E$ will just be the addition of all the (evolving) contributions of the source function between the beginning of the expansion, $t_{0}$, and $t$ (these contributions are given by Eq. (C.5)). The resulting integral is

$$
\begin{aligned}
& N(E, t)= \\
& \int_{t_{F}}^{t} \frac{S(\tau)(m-1-\rho)^{2-p} E^{-p}(\tau / t)^{m} \mathrm{~d} \tau}{\left((m-1-\rho)(\tau / t)^{m}+2 / 3 c_{2} B_{\mathrm{ref}}^{2} E t_{\mathrm{ref}}^{-\rho}\left((\tau / t)^{m} t^{\rho+1}-\tau^{1+\rho}\right)\right)^{2-p}} .
\end{aligned}
$$

\section{Appendix D: Why are the light curves flatter if we consider radiative cooling?}

From Eq. (3) we can approximate the value of $\beta$ by assuming that at any time the electron energy distribution does not differ so much from the canonical one, $N \propto E^{p}$. Under this assumption (valid for a rough qualitative discussion), we can show that

$\beta=\frac{\mathrm{d} \ln (I)}{\mathrm{d} \ln (t)} \propto \frac{\dot{I}}{I}=\frac{1+p}{2} \frac{\dot{B}}{B}+\frac{\dot{N}}{N}$.

The term with $\dot{B} / B$, which is negative and is related to the different electron emissivities under different magnetic fields, has the same effects on $\beta$ whether radiative cooling is considered or not. With regard to the term $\dot{N} / N$, which is positive and is related to the different total emissivity for different number of electrons, when radiative cooling is not considered it takes the form

$\frac{\dot{N}(E, t)}{N(E, t)}=\frac{S(E, t)}{\int_{t_{0}}^{t} S\left(E, t^{\prime}\right) \mathrm{d} t^{\prime}}$,

where $S(E, t)$ is the source function given in Eq. (7). However, if radiative cooling is considered, we have instead

$\left(\frac{\dot{N}(E, t)}{N(E, t)}\right)_{\mathrm{Cool}}=\frac{S(E, t)-c(E, t)}{\int_{t_{0}}^{t}\left(S\left(E, t^{\prime}\right)-c\left(E, t^{\prime}\right)\right) \mathrm{d} t^{\prime}}$, where $c(E, t)=-\nabla_{E}(N \dot{E})$ and $\dot{E}$ is given in Eq. (10). The function $c(E, t)$, which indirectly depends on $N$, approaches 0 as time goes by. Therefore, the numerator, $\dot{N}(E, t)$, if cooling is considered, will tend to that of the cooling-free case, while the denominator, $N(E, t)$, will always be smaller in the case with cooling. Hence,

$\left(\frac{\dot{N}(E, t)}{N(E, t)}\right)_{\mathrm{Cool}}>\frac{\dot{N}(E, t)}{N(E, t)}$.

From this equation, it is easy to see that the positive contribution to $\beta$ provided by $\dot{N} / N$ (see Eq. (D.1)) is larger if radiative cooling is considered. The corresponding value of $\beta$ is, therefore, closer to 0 (the light curve is flatter).

The inequality in Eq. (D.2) may have important observational effects until very late times (several years), when we will have

$\int_{t_{0}}^{t} S\left(E, t^{\prime}\right) \mathrm{d} t^{\prime} \gg \int_{t_{0}}^{t} c\left(E, t^{\prime}\right) \mathrm{d} t^{\prime}$.

Even then, the average value of $\beta$ for the whole optically-thin part of the light curve may still differ from that of the coolingfree case.

\section{References}

Alberdi, A., Colina, L., Torrelles, J. M., et al. 2006, ApJ, 368, 938 Bartel, N., \& Bietenholz, M. F. 2003, ApJ, 591, 301

Bartel, N., Bietenholz, M. F., Rupen, M., et al. 2002, ApJ, 581, 404 Bietenholz, M. F., Bartel, N., \& Rupen, M. P., et al. 2002, ApJ, 581, 1132 Bietenholz, M. F., Bartel, N., \& Rupen, M. P., et al. 2004, Science, 304, 1947

Bietenholz, M. F., Bartel, N., \& Rupen, M. P., et al. 2010, ApJ, 712, 1057

Brunthaler, A., Martí-Vidal, I., Menten, K. M., et al. 2010, A\&A, 516, A27

Chandra, P., Dwarkadas, V. V., Ray, A., et al. 2009, ApJ, 699, 388

Chevalier, R. A. 1982a, ApJ, 258, 790

Chevalier, R. A. 1982b, ApJ, 259, 302

Colina, L., Alberdi, A., Torrelles, J. M., et al. 2001, ApJ, 553, L19

Ferrarese, L., Freedman, W. L., Hill, R. J., et al. 1996, ApJ, 464, 568

Fransson, C., \& Björnsson, C.-I. 1998, ApJ, 509, 861

Gull, S. F. 1973, MNRAS, 161, 47

Jun, B.-I., \& Norman, M. L. 1995, Ap\&SS, 233, 267

Marcaide, J. M., Pérez-Torres, M. A., Ros E., et al. 2002, A\&A, 384, 408

Marcaide, J. M., Martí-Vidal, I., Alberdi, A., et al. 2009a, A\&A, 505, 927

Marcaide, J. M., Martí-Vidal, I., Pérez-Torres, M. A., et al. 2009b, A\&A, 503, 869

Marchili, N., Martí-Vidal, I., Brunthaler, A., et al. 2010, A\&A, 509, A47

Martí-Vidal, I. 2008, Ph.D. Thesis, University of Valencia

Martí-Vidal, I., Marcaide, J. M., Alberdi, A., et al. 2011a, A\&A, 526, A142

Martí-Vidal, I., Marcaide, J. M., Alberdi, A., et al. 2011b, A\&A, 526, A143

Mioduszewski, A. J., Dwarkadas, V. V., \& Ball, L. 2001, ApJ, 562, 869

Muxlow, T. W. B., Beswick, R., Pedlar, A., et al. 2009, Atel, 2073

Muxlow, T. W. B., Beswick, R., Garrington, S. T., et al. 2010, MNRAS, 404, L109

Nymark, T. K., Chandra, P., \& Fransson, C., et al. 2009, A\&A, 494, 179

Pacholczyk, A. G. 1970, Radio Astrophysics (San Francisco: Freeman)

Pérez-Torres, M. A., Alberdi, A., \& Marcaide, J. M. 2001, A\&A, 374, 997

Pérez-Torres, M. A., Alberdi, A., Colina, L., et al. 2009, MNRAS, 399, 1641

Sanders, D. B., Mazzarella, J. M., Kim, D.-C., et al. 2003, AJ, 126, 1607

Tonry, J. L., Dressler, A., Blakeslee, J. P., et al. 2001, ApJ, 546, 681

Weiler, K. W., Panagia, N., \& Sramek, R. A. 1990, ApJ, 364, 611

Weiler, K. W., Van Dyk, S. D., Panagia, N., et al. 1991, ApJ, 380, 161

Weiler, K. W., Panagia, N., Montes, M. J., \& Sramek, R. A. 2002, ARA\&A, 40 387

Weiler, K. W., Williams, C. L., Panagia, N., et al. 2007, ApJ, 671, 1959 